\title{
Functional ability and nutritional status of free-living elderly people
}

\author{
BY MARY C. MANANDHAR \\ Human Nutrition Unit, London School of Hygiene and Tropical Medicine, 2 Taviton Street, \\ London WC1H OBT
}

HEALTH AND FUNCTION IN AN AGEING WORLD

In 1990 almost half a billion people in the world were over 60 years old (World Bank, 1994). In only 40 years time this number will nearly triple. By the end of those same 40 years three-quarters of the world's elderly population will be living in developing countries (Kinsella \& Suzman, 1992). The challenge for the next century is to alter focus accordingly.

Living longer comes at a price. Extra years will not necessarily be years of good health. In the complicated context of ageing biology, transitions from disease to disability rather than disease to death take on prime importance for older people. For many chronic diseases that can never be completely cured, delaying the onset of functional decline, or minimizing the impact of lost independent function on daily life, in effect approximates a 'cure' of the condition for those concerned (Besdine, 1990). Moreover, the presence of illness as measured by objective clinical data does not necessarily determine the presence or severity of dependency. And of course for many elderly people in developing countries the luxury of access to such knowledge is often unavailable. With risk of death increasingly likely, and both the long- and short-term effects of disease, diet and life style hard to disentangle, the traditional health measures of life expectancy and morbidity begin to appear inadequate outcomes against which to measure any health or nutritional indicator in older people, or evaluate preventive strategies. To what then should we turn?

The present paper addresses the following questions: Is functional ability an appropriate outcome indicator of health and nutritional status in free-living people in developing countries? What is functional ability and how can it be assessed? Is there any evidence of a relationship between functional ability and nutritional status in free-living elderly people? I will focus on the community as the site in which functional impairment first appears most often and my bias is towards malnutrition in developing countries. At times, I will refer to our own recent fieldwork experience amongst elderly slum dwellers in Bombay, but no data will be presented. The present paper aims to serve as an introduction to a new issue of growing practical importance in both nutritional gerontology and nutrition in the Third World.

\section{FUNCTIONAL ABILITY AS AN OUTCOME INDICATOR OF HEALTH IN OLD AGE}

Functional ability is the ability to perform basic activities of daily life without support, which is the key to overall independence and quality of life. We must be able to identify and measure factors limiting functional ability. We need to find ways of identifying people most at risk of losing these abilities (Skelton et al. 1994). 
The terms independence and quality of life may not mean the same, or have the same value in all cultures. Nevertheless, preserving functional ability is of particular importance for elderly people and their families in developing countries, where they often make a significant contribution to family welfare and income by their involvement in tasks such as child care, thus freeing younger adults for wage-earning. Many also continue to contribute to the family income well into old age. Continued participation of elderly people in the daily life of the household will depend on their level of functional ability. It may well also affect the way they are treated and respected. There is evidence that in many developing countries, especially in the growing urban areas, the extended family and traditional coping systems for older people are beginning to show signs of strain (Hashimoto et al. 1992). Thus, understanding the mechanisms behind the maintenance of functional ability, and devising strategies to preserve it for as long as possible, will have a beneficial impact on millions of elderly people and their families in a number of physical, economic, social and emotional ways.

The passage from a state of independence to that of dependence is characterized by the inability to perform activities of daily living such as getting out of bed, dressing, personal hygiene, eating and walking. Functional ability refers only to that part of functional capacity which is related to essential activities of daily life. Functional ability impairment means a decreased ability to meet one's own daily needs (Besdine, 1990).

Functional ability assessment summarizes the net impact of pathobiological processes and morbid conditions, many of which are still incompletely understood in old age (Wallace \& Rohrer, 1990). It can be applied at both individual and population levels to obtain needs assessment information and assist in some subsequent course of action, such as assigning goals and monitoring progress. Functional ability assessment has long been the cornerstone of rehabilitation. With population ageing, we might argue that it should soon become the cornerstone of public health. Many instruments for functional ability assessment have been developed, none of which are particularly refined, easily conducted or the results interpreted. They have been extensively reviewed (Kane \& Kane, 1981; Branch \& Meyers, 1987), and are not discussed in detail here. Instead, the emphasis is placed on two main types of assessment techniques, and the dimensions they claim to measure.

\section{ASSESSING FUNCTIONAL ABILITY BY SELF-REPORT: ACTIVITIES OF DAILY LIVING (ADL) AND INSTRUMENTAL ADL (IADL)}

Self-reporting is the most prevalent type of functional ability assessment in geriatric programmes and research, with a long history and wide applications (Liang \& Jette, 1981). It involves merely asking subjects (or family member and/or carer in proxy reporting) whether they are capable of performing a task by themselves or with assistance. Two main types of self-report instruments have been developed. First, an ADL self-report questionnaire assesses basic mobility and essential self-maintenance skills. About 30 years ago, Katz et al. (1963) demonstrated that the sequence of recovery of six 'basic' activities of daily living among disabled elderly (i.e. bathing, dressing, toileting, transferring, continence and feeding) resembled the sequence of development of these same functions in child development across the spectrum of human societies (Katz \& Stroud, 1989). In developing and testing their index of ADL, the team not only first facilitated the measurement of functional ability, but also channelled our minds to 
the importance of this outcome in the context of daily life (Spitzer, 1987). Their original work has now been expanded and developed so much that for any research or programme involving the elderly the ability to perform ADL has become a standard variable in the analyses (Weiner et al. 1990). The second type is the IADL self-report questionnaire which assesses housekeeping, shopping, handling money and wider mobility such as using public transport. Developed a few years after the Katz et al. (1963) ADL by Lawton \& Brody (1969), the IADL instrument deals with more complex activities relevant to a minimally adequate social life. IADL are more sensitive in detecting modest functional loss than ADL, and have since also been modified and revised. Later research suggested that a hierarchical relationship exists between some ADL and IADL items, and a combined scale with high discriminant and predictive validity was recommended (Spector et al. 1987).

There are over 200 published indices that assess ADL and IADL, the majority of which have not been adequately evaluated (Shah et al. 1989). The assumptions and constraints of ADL and IADL have been addressed by a number of authors (Kane \& Kane, 1981; Liang \& Jette, 1981; Fillenbaum, 1984; Feinstein et al. 1986; Guralnik et al. 1989; Law \& Letts, 1989). They include insensitivity to changes in health status, the assumption 'if you can you will' which is not necessarily true in all cases, the subjectivity of many responses and potential inaccuracy for reasons of language, comprehension, hearing, intellectual capacity, level of motivation and cooperation, or even pride (Rodgers \& Herzog, 1987). But the biggest problem is lack of adequate validation. However, the general consensus on self-reports is that they are deemed to give a reasonable approximation, obtained relatively quickly. There is even evidence that they identify more accurately levels of functional impairments in elderly people than do clinical or nursing judgements (Pinholt et al. 1987).

\section{ASSESSING FUNCTIONAL ABILITY BY PHYSICAL PERFORMANCE TESTS}

Acknowledgement of the constraints in these self-report instruments has led to the development of physical tests which represent more objective and quantifiable performance measures. These are thought to add more appropriate information on overall functional ability as they simulate some physical attributes essential to the satisfactory performance of activities of daily living, and offer the potential to overcome some of the limitations of self-reports (Guralnik et al. 1989; Reuben \& Siu, 1990). Functional ability has numerous dimensions or components and its assessment must be similarly constructed (Branch \& Meyers, 1987). Thus, physical performance tests can be described according to these different dimensions, i.e.: mobility, manual ability, flexibility, muscular strength, psychomotor and cognitive function. Evidence of correlations between these dimensions, as well as with other variables, can be found in the literature and will not be discussed here.

\section{Mobility}

The ability to get around in one's environment is a basic human function necessary for independence and activities of daily life (Wolfson, 1992). Loss of mobility is a principal cause of a limited quality of life and increased dependence in elderly people, especially for women. Descriptions of mobility performance tests in elderly people can be found in 
the reports of Tinetti (1986) and Podsiadlo \& Richardson (1991). Lower body dysfunction will affect mobility, and have an impact on other factors such as anxiety, fear of falls, and balance (Tinetti et al. 1994).

Mobility impairment can affect nutritional status by impeding participation in food production, acquisition, and preparation as well as in socialization of eating patterns. Conversely, nutritional factors have been implicated in motor and gait disorders (vitamin $\mathrm{B}_{12}$ deficiency), osteoporosis (longstanding $\mathrm{Ca}$ deficiency, and possibly vitamin $\mathrm{D}$ deficiency) and in precipitating fractures in subjects with a low bone-mineral density (Calkins, 1992; Department of Health, 1993), which in turn influence mobility, balance and proneness to falls (Vellas et al. 1992). Mansell et al. (1990) reported that low body weight and mid-arm circumference measurements were significantly associated with an increased risk of fractured neck of femur and of vertebral collapse in three groups of elderly women, including those living in the community. Bastow et al. (1983) highlighted the importance of the increased risk of hypothermia in thin undernourished elderly people because of its impact on lack of coordination, accident and mobility.

\section{Manual ability}

The ability to use one's hands proficiently in everyday activities is required for the majority of ADL skills. A decrement in hand function appears to be a significant musculoskeletal mechanism causing disablement in basic ADL and may be one of the earliest markers of impending functional decline (Jette et al. 1990). Several versions of a simple timed manual performance test have been developed by Williams et al. (1982, 1994), which have proved to be highly correlative and predictive markers of functional dependency, both in the institutional setting and in the community (Ostwald et al. 1989; Mowat et al. 1992). The test battery involves the performance of commonplace tasks such as opening fasteners, simulating eating, picking up small objects and copying a simple sentence. However, some of these may be inappropriate for use in other cultures. In the Bombay slums, the elderly population is largely illiterate, and the wide variety of fasteners unfamiliar, so we used only a simple lock and key test in our study. Older people suffering from rheumatoid arthritis may find fine neuromuscular control and coordination so reduced that it is difficult to hold or use utensils, procure food, prepare it safely or even self-feed. Such people frequently have a thin emaciated appearance, attributed in part to these manual problems but also to difficulties in chewing or swallowing because of arthritis affecting the jaw, and to chronic gastritis secondary to medication (Calkins, 1992).

\section{Flexibility and range of motion}

Decreases in flexibility also typically occur with advancing age, mainly because joints become stiff and painful, articulating surfaces are damaged, range of motion is reduced and there is accompanying gradual lack of use (Badley et al. 1984; Bassey et al. 1989). The individual's ability to undertake daily activities is consequently reduced. Simple, reliable and socially acceptable performance tests, such as the earlobe test (Bergstrom et al. 1985), posterior reach (Rikli \& Busch, 1986) and shoulder abduction (Bassey, $1990 \mathrm{~b}$; used in Bombay), can assess flexibility and range of motion in elderly people living in the community. These tests have functional relevance as they simulate 
important movements in activities of daily life, such as dressing and grooming, hanging out washing, and using shelves, hooks or supporting rails. Deterioration in this dimension has been linked to reduced independence (Jette et al. 1990).

There is some evidence to suggest a relationship between nutritional status and range of motion in elderly people, but it is inconsistent. A Swedish study found that so-called 'lean' subjects reported more shoulder problems, more restricted range of motion and increased ADL assistance than so-called 'non-lean' individuals (Bergstrom et al. 1985). However, Bassey et al. (1989) reported that BMI was significantly negatively related to shoulder range in women. They hypothesized that compression of fatty tissue will affect stiffness of joints and limit joint range, particularly in the presence of low muscle strength.

\section{Muscular strength}

By age 70 years it is estimated that muscle strength is about $35-45 \%$ lower than its peak value in youth, although the decline varies according to muscle group and sex (Grimby \& Saltin, 1983).

Physical performance tests of the muscular strength dimension of functional ability in older adults have concentrated mainly on isometric tests of handgrip and quadriceps muscle (knee extension and flexion). These have all been found in young Caucasian adult males to relate well to aggregate measures of muscle strength (Bassey, 1990a). The power grip of the hand requires the combined action of a number of muscles in both the hand and forearm, and is a familiar action of functional importance for many activities of daily life (transferring tasks, holding onto supports, opening containers, using tools, dressing). As our Bombay experience showed, it can be measured easily in the field setting, preferably using a strain-gauge dynamometer (Bassey, 1990a). Low handgrip strength implies considerable functional disadvantages (Kallman et al. 1990; Bassey \& Harries, 1993). Handgrip strength is more sensitive than body composition measurements to predict post-operative complications and mortality (Klidjian et al. 1980; Webb et al. 1989).

Quadriceps muscle strength is also of great functional significance in daily activities, but progressively decreases from the third decade onwards (Rutherford \& Jones, 1992; Skelton et al. 1994). Lack of strength in this muscle will affect most weight-bearing activities, general locomotion and mobility, independence in transferring (Bergstrom et al. 1985) and proneness to falls and fractures (Bastow et al. 1983; Nevitt et al. 1989; Wickham et al. 1989). Whilst laboratory-based studies use fixed power-rigs or chair dynamometers to measure quadriceps strength, community studies can use the ability (time taken) to rise repeatedly from a chair without the use of hands. This was developed as a simple standardized test relevant to activities of daily life that can indicate the level of lower body strength and which correlates strongly with the rig or chair methods (Csuka \& McCarty, 1985; Alexander et al. 1991; Bassey et al. 1992). Chair stands may not be such an appropriate test for populations who use neither chairs nor beds in daily life, but who sit and sleep on the floor. In Bombay, we struck an additional complication. As chair rises were used as a punishment in schools, some subjects were not initially keen to perform them.

What effect does this loss of muscular strength have on the functional ability of community-living elderly people? Professor Archie Young $(1986,1992)$ introduced the 
concept of threshold, and the narrowing of safety margins between normality and abnormality of function. As one grows older there comes a time when maximal strength in a particular action is the same as the minimum required to perform an everyday activity. A gradual loss of strength may not be apparent until the person is suddenly unable to perform a crucial function, or is at least so dependent on aerobic metabolism as to render it unpleasant to perform. Assuming a rise time of less than $3 \mathrm{~s}$, Young (1986, 1992) speculated that a healthy 80 -year-old woman is at, or very near to, the threshold value of quadriceps strength for rising from a low armless chair or lavatory seat. We might infer that an unhealthy, malnourished old woman may experience this threshold some years earlier. A recent paper by Skelton et al. (1994) represents the first major analysis of muscle strength and power and their relationship to functional ability in old age. The authors caution that the relationship between even laboratory measures of physical performance and functional ability is still poorly understood. Associations between functional ability and strength and power have been demonstrated in the presence of pathology, but more research is needed in this area, especially with regard to those performance tests suitable for use in the community. Muscular strength presumably affects nutritional status by its impact on mobility, physical activity level and energy expenditure, as well as basic functional ability tasks such as transferring (Rantanen et al. 1994). More will be said a little later about the impact of nutritional status on this muscular strength dimension of functional ability.

\section{Psychomotor and cognitive function}

The ability of an individual to process, and react to, specific external information is considered an important indicator of overall cerebral status (Panton et al. 1990). Decline in psychomotor function is age-related (Fozard et al. 1994). Deterioration of this dimension of functional ability will jeopardize capacity to cope with independence in $\mathrm{ADL}$ and IADL, although most research has focused on indirect cross-sectional investigations on age, physical fitness and psychomotor speed (Spirduso, 1980; Rikli \& Busch, 1986). Simple and choice reaction times, tapping speed and a plate-tapping test (Bassey, 1990b; used successfully in Bombay) can be used to measure the speed and coordination skills of this dimension in elderly people in community studies. Evidence of a nutritional association with psychomotor function in elderly people has not been established.

Some assessment of mental status and cognitive function is necessary in any multidimensional assessment of functional ability to act as a screen in identifying those individuals incapable of understanding test instructions and whose responses about ADL and IADL may also be suspect. Instruments for assessing cognitive aspects of mental function in the aged vary greatly in content, detail and administration time (Little et al. 1987), although probably the most well-recognized and validated is the Folstein $e t$ al. (1975) mini-mental state examination, a scored series of eleven questions concerned with orientation, memory, attention and the ability to follow verbal and written commands. It has a high reliability, is significantly correlated with more sophisticated tests of neuropathology, and distinguishes between people with or without cognitive disturbances (Folstein et al. 1975, 1985).

As there are behavioural components to all determinants of diet and nutritional status, it follows that for elderly people cognitive status plays a crucial role in nutritional status, 
which in turn will affect physical activity and behaviour. Conversely, the role of proper nutrition on cognitive function may also be very important. Whilst this is well appreciated in early brain development, evidence of nutritional influences on later cognitive function and mental performance in older adults is more controversial (Evans, 1994). Some work is suggestive of a relationship between nutritional status and cognition in elderly people. Epidemiological investigations have linked malnutrition with an increased risk of Alzheimer's disease, and some dietary deficiencies of vitamins $\left(C, B_{12}\right.$, thiamin, folate and riboflavin), and malabsorption of vitamin $B_{12}$ have all been implicated in cognitive dysfunction, especially in association with infection and other stresses (Goodwin, 1989). However, it is not clear whether sharper elderly people eat better, or better nutrition aids cognition, or some other undetermined factor is responsible for both (Roe, 1986). Poor cognition was significantly associated with low $\mathrm{BMI}$ and weight loss in studies of elderly nursing home residents in seven American states (C. S. Blaum, B. E. Fries and M. A. Fiatarone, unpublished results). Despite the limitations of correlational data, the hypothesis that poor nutritional status can contribute to neurocognitive decline in otherwise healthy individuals is intriguing (Evans, 1994).

\section{Constraints of physical performance tests of functional ability}

Constraints of these tests include space, time, staff and props requirements, motivational skills of the examiner, and motivation, comprehension and compliance of the subject (Martin et al. 1985; Shizgall et al. 1986; Bassey \& Harries, 1993). Pain, stiffness, fatigue, intimidational and other psychosocial factors might influence test performance in elderly people. Physical risks may also be involved, such as falling or angina (Wallace \& Rohrer, 1990). Large variation between normal individuals has been found in some tasks, such as handgrip strength. Attributes of reliability, validity and sensitivity are undocumented for many tests, and the ability of most to predict functional decline is unproven (Reuben \& Siu, 1990). Results of such tests should probably still be interpreted with caution until there is better understanding of relationships between performance and self-reports, and they have been used in other populations (Guralnik et al. 1989).

\section{COMBINING PERFORMANCE TESTS AND SELF-REPORTS}

Despite these constraints, performance tests are already routinely combined into batteries of tests for use in the clinical setting as part of physical and occupational therapy. However, they are still not widely incorporated into the assessment of free-living elderly, or for population-based research. Batteries of simple performance tests are now being utilized in some surveys in Europe (Bassey, 1990b), Scandinavia (Heikkinen et al. 1993) and the USA including the US National Health and Nutrition Examination Survey III. Branch \& Meyers (1987) and Guralnik et al. (1989) reviewed various instruments involving performance-based ADL. In a longitudinal study of elderly people in different situations, significant independent predictive effects for ADL, gait score and seven-item physical performance test on death and nursing home placement were found (Reuben \& Siu, 1990). Recently, Ensrud et al. (1994) explored factors associated with impaired function in community-living older women by utilizing both ADL and IADL, and some physical performfance tests as well as health, 
anthropometry, sensory status and habits such as alcohol drinking and smoking. The rationale for their study was that the association between impaired function and modifiable risk factors had not been adequately studied.

The wider use of objective measures of functional ability to supplement the traditional self-report measures in parallel with classic disease-orientated techniques is recommended (Guralnik et al. 1989; Besdine, 1990; Reuben et al. 1992). However, there is as yet no 'gold standard' for overall functional ability. Although Siu et al. (1993) used their physical performance test and other instruments to test the validity of self-reports, they acknowledged that the performance measures do not themselves form any sort of gold standard for the dimensions involved. Branch \& Meyers (1987) likened the search for the right functional ability assessment instrument to the search for the holy grail, but remain optimistic: 'Slow progress notwithstanding, the search will continue for a reliable and valid all-purpose instrument, one that summarises physical functioning status into a simple page and can be summarised further into a single score, and that will be useful in just about all health care contexts'. We must ensure that this includes community use in developing countries.

FUNCTIONAL ABILITY AND NUTRITIONAL STATUS: ARE THEY RELATED?

Calls for research on the establishment of associations between physical functional ability and other indicators have been increasing in recent years (Branch \& Meyers, 1987; Guralnik et al. 1989; Katz \& Stroud, 1989; Rosenberg \& Miller, 1992). We might begin by asking to what extent nutritional status influences functional dependence in elderly people? In the institutional setting there is evidence that proper evaluation of an elderly patient's nutritional status is important. A significant correlation between measures of nutritional status and risk of developing major complications as well as mortality were found in a study of elderly admissions to a geriatric hospital (Sullivan et al. 1990). The nutritional impact on outcome was also found to be independent of all the other non-nutritional variables known to modulate morbidity and mortality. Non-nutritional factors of importance included functional status.

But what about elderly people still living in the community? Unfortunately, evidence of nutritional effects on functional ability, or vice versa, for community-living elderly people, are largely unavailable (Institute of Medicine, 1990; Department of Health, 1993; Payette, 1994).

Some major studies on nutrition in the elderly either did not include any functional ability assessments (e.g. Boston Nutritional Status Survey (Hartz, 1992)) or only included self-reports but no physical performance measures (e.g. EURONUT SENECA (De Groot \& Van Staveren, 1988)). The International Union of Nutritional Sciences is currently conducting studies on food habits and health status with a protocol that includes self-reported ADL and mental status assessment but no tests of physical performance of functional ability (Wahlqvist et al. 1994). The mini-nutritional assessment for elderly people being developed in Toulouse incorporates some physical performance measures such as grip strength, but full results are not yet available (Guigoz et al. 1993). The UK nationwide survey on elderly people being conducted by the Ministry of Agriculture, Fisheries and Food, and the Ministry of Health (Hughes et al. 1995 ) includes grip strength but no other physical performance tests of functional ability. Many surveys of functional ability in the elderly have included health but not nutritional 
assessments (Heikkinen et al. 1983). More thorough means of assessing ADL and IADL with special attention to nutrition are needed. Work on questionnaires to assess aspects of functional status that are associated with nutritional risk is apparently under way (Institute of Medicine, 1990).

Does nutritional status influence the muscular strength dimension of functional ability for elderly people? Malnutrition can certainly predispose to impairment of the muscular strength dimension of functional ability if weight loss reflects the effects of loss of lean body mass. Mowé et al. (1994) found that handgrip strength was significantly lower in a recently hospitalized and malnourished group of elderly, compared with a normallynourished home group. Studies on another muscle, the adductor pollicis muscle of the hand, may throw some light on the question of muscle strength and nutritional status. Shizgall et al. (1986) found that in malnourished (non-elderly) patients the muscle generated less force, fatigued faster and recovered slower than in normally-nourished patients, and concluded that the key factor is probably the availability of energy stores within the muscle and the ability to regenerate them from available substrate. Working with elderly subjects, Phillips et al. (1992) concluded that muscle atrophy and reduced force per cross-sectional area mean that older subjects have to use a higher proportion of their muscle maximal voluntary force to produce the same force as a younger subject. Roe (1986) recommended mid-arm circumference and triceps skinfold to calculate arm muscle area and detect wasting in elderly people, and believed that circumference measurements can be used to indicate loss of muscle mass and its functional consequences. Other nutritional factors relating to muscular strength have been documented, such as correlations between dietary $\mathrm{Mg}$ and muscle capacity, and circulating vitamin $\mathrm{D}$ and muscle strength (Institute of Medicine, 1990).

In a lecture recently delivered at the London School of Hygiene and Tropical Medicine, Professor Nevin Scrimshaw stated that 'almost any indicator of function correlated with BMI shows those with low BMI to be worse off'. But there is no evidence that this holds true for community-living elderly people in terms of their functional ability outcomes. Indeed, there is evidence to the contrary, although this probably reflects the lack of data on nutritional status and functional ability for elderly populations where low BMI are more prevalent. A high BMI was a strong independent risk factor for impaired function (reported difficulty in performing three or more ADL) in a large study of ambulatory old women in the USA (Ensrud et al. 1994), and was also found to be strongly associated with physical performance tests in a recent analysis of three large community-based studies of elderly in the USA (Seeman et al. 1994). However, it is important to remember that BMI should be used and interpreted with caution in any nutritional assessment of elderly people (James et al. 1988).

The Framingham Disability Study raised important questions relating to functional ability and nutritional status. For example, does relative weight have an influence on elderly people's ability to perform basic life activities (Jette \& Branch, 1981)? An analysis of the American Longitudinal Study of Aging found that cerebrovascular disease, arthritis and possibly coronary artery disease increased the likelihood of functional limitations (as measured by ADL and IADL), but not abnormally high weights (Boult et al. 1994). But what about free-living populations where low weights are more prevalent? The data are not available. A group in the USA found a strong association between feeding dependency and poor intake and both low BMI and weight loss but only in nursing home residents (C. E. Blaum, B. E. Fries and M. A. Fiatarone, 
unpublished results). Underweight older subjects certainly have decreased reserve capacity and can develop serious nutritional problems both rapidly and with only minor stress. Malnutrition is probably responsible for many non-specific symptoms observed in elderly people, such as chronic fatigue, general feeling of ill-health and loss of appetite, which will eventually lead to more pronounced malnutrition. In the USA there is substantial evidence of specific nutritional deficiencies in independent elderly (based on intakes falling below recommended daily allowances; Goodwin, 1989), but it is not at all clear how functionally important such findings are. So although it thus seems sensible to conclude that malnutrition in elderly people can lead to problems related to independent functional ability and social integration, a large body of quantifiable evidence still eludes us.

A number of other variables affect mobility, manual dexterity and degree of socialization, and can thus be expected to have an impact on both nutritional status and functional ability in elderly people living in the community. Many such factors are reviewed in the literature (Goodwin, 1989; Lehmann, 1989; Davies \& Knutson, 1991; Grimby et al. 1993) and include social isolation, depression, problems with dentition and swallowing, fear of incontinence, and declining sensory abilities. These all influence food acquisition, preparation, choice and level of intake, as well as socialization of eating patterns, and may lead to a tendency to neglect food.

\section{THE STATE OF THE ART: RESEARCH PRIORITIES}

I have failed to find any published study that directly assesses nutritional status and functional ability of free-living elderly in a developing country, and few papers that covered even one of these aspects. What can be done to address this? In general, we need more research on anthropometric and body composition indicators of nutritional status and their interactions and associations with functional ability in elderly people. A number of reviews on nutritional assessment of the elderly outline the undoubted theoretical and practical problems involved especially with regard to anthropometry and body composition (Roe, 1986; Chumlea \& Baumgartner, 1989; Lehmann, 1989; Kelly \& Kroemer, 1990; World Health Organization Subcommittee on Anthropometry in the Elderly, unpublished results). However, such problems are no excuse for inaction. More research is also needed in relation to vitamin and mineral status indicators and functional ability outcomes.

In the Western world the elderly are probably the largest population group at risk of undernutrition. In the developing world the risk will increase as surely as the numbers will grow. But there is an alarming lack of data on the nutritional status of free-living elderly people from developing countries. The childhood bias in nutritional research has much to answer for, as we face a situation where the least knowledge exists for that very section of the world's population projected to increase the most and where institutional care is largely unfamiliar, but where traditional systems of elderly care and support are increasingly under threat.

A similarly poor situation exists for functional ability. Protocols incorporating functional ability assessments are only just beginning to be assembled in developing countries. There have been very few published applications of performance-based measures of physical function in developing countries, although a number of efforts are either under way or being planned (Guralnik et al. 1989; Fillenbaum, 1990). The World 
Health Organization has been increasingly active in this field, especially through its Special Program on Aging, of which a community study in Sri Lanka is a recent example (Fernando \& Seneviratna, 1993).

We urgently need validations of functional ability assessment techniques (both self-report and physical performance tests) from developing countries. The appropriateness of some existing instruments of functional ability assessment to non-Western cultures may be questionable. In our own study of elderly people in Bombay slums we found, as have others, that an elderly person's cultural environment may not encourage or expect a certain behaviour (covered in an ADL or IADL question, or physical performance test), according to norms of social and economic status, age, sex and prestige (Syryani et al. 1988; Barker, 1989). Existing instruments for IADL are especially problematic, and often gender-specific. Many ADL tasks are performed in quite different ways in non-Western cultures (Jitapunkul et al. 1994). Barker (1989) remarked especially on the problem of such populations simply being unused to answering direct probing questions about their lives. Conducting physical performance tests of functional ability in our Bombay slums study we found adaptations were necessary due to constraints of space, light and locally-available equipment. However, elderly subjects cooperated in all tests, many enjoying the activities as a change from their normal daily routine. Our experience confirms that functional ability assessments, including performance tests, can be implemented in the field, although their interpretation should probably still be undertaken with some caution (Solomons, 1992).

\section{CONCLUSION}

For all age-groups, including the elderly, tackling the causes of malnutrition must be our ultimate goal. However, being able to measure and monitor nutritional status in relation to a functional outcome of practical importance is imperative. Consequently, nutritional assessment for the elderly must be orientated towards providing useful information on the transitions to functional ability impairment. We must begin by identifying in more precise ways the manner in which nutritional status contributes to the preservation or deterioration of functional abilities in older people, and vice versa. Some evidence suggests that nutritional status is important for the maintenance of functional ability and independence for an older adult. We may not have convincing evidence of the relationship, but that does not mean the question is unimportant, rather that more information is needed. It could suggest possible ways in which intervention might restore either in the elderly. Whilst some progress is being made in Western countries, the collection of even basic data on nutrition and functional ability amongst free-living elderly people in developing countries has hardly begun. Given the emerging demographic phenomenon of ageing predominantly facing those countries, there is little time to waste.

The author is funded by HelpAge International. The collaboration of Dr Suraiya Ismail (London) and Mrs P. S. Anklesaria (Bombay), and the support of colleagues at HelpAge International, HelpAge India, and the Seth GS Medical College and King Edward VII Memorial Hospital in Bombay are gratefully acknowledged. 


\section{REFERENCES}

Alexander, N. B., Schultz, A. B. \& Warwick, D. N. (1991). Rising from a chair: effects of age and functional ability on performance biomechanics. Journal of Gerontology 46, M91-M98.

Badley, E. M., Wagstaff, S. \& Wood, P. H. N. (1984). Measures of functional ability (disability) in arthritis in relation to impairment of range of joint motion. Journal of Gerontology 43, 563-569.

Barker, J. C. (1989). Health and functional status of the elderly in a Polynesian population. Joumal of Cross-Cultural Gerontology 4, 163-194.

Bassey, E. J. (1990a). Tests of muscle strength. In Handbook of Methods for the Measurement of Work Performance, Physical Fitness and Energy Expenditure in Tropical Populations, pp. 59-65 [K. J. Collins, editor]. London: International Union of Biological Sciences, Medical Research Council and London School of Hygiene and Tropical Medicine.

Bassey, E. J. (1990b). Simple performance tests. In Handbook of Methods for the Measurement of Work Performance, Physical Fitness and Energy Expenditure in Tropical Populations, pp. 67-79 [K. J. Collins, editor]. London: International Union of Biological Sciences, Medical Research Council and London School of Hygiene and Tropical Medicine.

Bassey, E. J., Fiatarone, M. A., O’Neill, E. F., Kelly, M., Evans, W. J. \& Lipschitz, L. A. (1992). Leg extensor power and functional performance in very old men and women. Clinical Science 82, 321-327.

Bassey, E. J. \& Harries, U. J. (1993). Normal values for handgrip strength in 920 men and women aged over 65 years, and longitudinal changes over 4 years in 620 survivors. Clinical Science 84, 331-337.

Bassey, E. J., Morgan, K., Dallosso, H. M. \& Ebrahim, S. B. J. (1989). Flexibility of the shoulder joint measured as range of abduction in a large representative sample of men and women over 65 years of age. European Journal of Applied Physiology 58, 353-360.

Bastow, M. D., Rawlings, J. \& Allison, S. P. (1983). Undernutrition, hypothermia and injury in elderly women with fractured femur: an injury response to altered metabolism? Lancet i, 143-146.

Bergstrom, G., Aniansson, A., Bjelle, A., Grimby, G., Lundgren-Linquist, B. \& Svanborg, A. (1985). Functional consequences of joint impairment at age 79. Scandinavian Journal of Rehabilitative Medicine 17, $183-190$.

Besdine, R. W. (1990). Functional assessment in the elderly. In Geriatric Medicine, 2nd ed., pp. $37-51$ [J. L. Rowe and R. W. Besdine, editors]. Boston: Little, Brown and Co. Inc.

Boult, C., Kane, R. L., Louis, T. A., Boult, L. \& McCaffrey, D. (1994). Chronic conditions that lead to functional limitation in the elderly. Journal of Gerontology 49, M28-M36.

Branch, L. G. \& Meyers, A. R. (1987). Assessing physical function in the elderly. Clinics in Geriatric Medicine 3, 29-51.

Calkins, E. (1992). Management of rheumatoid arthritis in the elderly patient. In Oxford Textbook of Geriatric Medicine, pp. 356-362 [J. Grimley Evans and T. Franklin Williams, editors]. Oxford: Oxford University Press.

Chumlea, W. C. \& Baumgartner, R. N. (1989). Status of anthropometry and body composition data in elderly subjects. American Journal of Clinical Nutrition 50, 1158-1166.

Csuka, M. \& McCarty, D. J. (1985). Simple method for measurement of lower extremity muscle strength. American Journal of Medicine 78, 77-81.

Davies, L. S. \& Knutson, K. C. (1991). Warning signals for malnutrition in the elderly. Journal of the American Dietetic Association 91, 1413-1417.

Department of Health (1993). The Nutrition of Elderly People. Report on Health and Social Subjects no. 43. London: H.M. Stationery Office.

Ensrud, K. E., Nevitt, M. C., Yunis, C., Cauley, J. A., Seeley, D. G., Fox, K. M. \& Cummings, S. R. (1994). Correlates of impaired function in older women. Journal of the American Geriatrics Society 42, 481-489.

Evans, P. H. (1994). Nutrient and toxin interactions in neurodegenerative disease. Proceedings of the Nutrition Society 53, 431-442.

Feinstein, A. R., Josephy, B. R. \& Wells, C. K. (1986). Scientific and clinical problems in indexes of functional disability. Annals of Internal Medicine 105, 413-420.

Fernando, D. N. \& Seneviratna, R. de A. (1993). Physical health and functional ability of an elderly population in Sri Lanka. Ceylon Journal of Medical Sciences 36, 9-16.

Fillenbaum, G. G. (1984). The Well-being of the Elderly: Approaches to Multidimensional Assessment. WHO Offset Publication no. 84. Geneva: WHO.

Fillenbaum, G. G. (1990). Assessment of health and functional status: an international comparison. In Improving the Health of Older People. A World View, pp. 69-90 [R. L. Kane, J. Grimley Evans and D. Macfayden, editors]. New York: Oxford University Press and WHO. 
Folstein, M., Anthony, J. C., Parhad, I., Duffy, B. \& Gruenberg, E. M. (1985). The meaning of cognitive impairment in the elderly. Journal of the American Geriatrics Society 33, 228-235.

Folstein, M. F., Folstein, S. E. \& McHugh, P. R. (1975). Mini-Mental State: a practical method for grading the cognitive state of patients for the clinician. Journal of Psychiatric Research 12, 189-198.

Fozard, J. L., Vercruyssen, M., Reynolds, S. L., Hancock, P. A. \& Quilter, R. E. (1994). Age differences and changes in reaction time: the Baltimore Longitudinal Study of Aging. Journal of Gerontology 49, P179-P189.

Goodwin, J. S. (1989). Social, psychological and physical factors affecting the nutritional status of elderly subjects: separating cause and effect. American Journal of Clinical Nutrition 50, 1201-1209.

Grimby, A., Milsom, I., Molander, U., Wiklund, I. \& Ekelund, P. (1993). The influence of urinary incontinence on quality of life of elderly women. Age and Ageing 22, 82-89.

Grimby, G. \& Saltin, B. (1983). The ageing muscle. Clinical Physiology 3, 209-218.

De Groot, C. P. G. M. \& Van Staveren, W. A. (1988). Nutrition and the Elderly: Manual of Operations, Euronut report 11 . Wageningen, The Netherlands: Euronut.

Guigoz, Y., Vellas, B. \& Garry, P. J. (1993). The Mini-Nutritional Assessment: a practical assessment tool for grading the nutritional status of elderly patients. Facts and Research in Gerontology, Suppl. 2.

Guralnik, J. M., Branch, L. G., Cummings, S. R. \& Curb, J. D. (1989). Physical performance measures in aging research. Journal of Gerontology 44, M141-M146.

Hartz, S. C. (1992). Nutritional status survey: design and methodology. In Nutrition in the Elderly: The Boston Nutritional Status Survey, pp. 17-25 [S. C. Hartz, R. M. Russell and I. H. Rosenberg, editors]. London: Smith-Gordon/Nishimura.

Hashimoto, A., Kendig, H. L. \& Coppard, L. C. (1992). Family support to the elderly in international perspective. In Family Support for the Elderly, pp. 293-308 [H. L. Kendig, A. Hashimoto and L. C. Coppard, editors]. New York: Oxford University Press and WHO.

Heikkinen, E., Era, P., Jokela, J., Jylha, M., Lyyra, A. \& Pohjolainen, P. (1993). Socioeconomic and lifestyle factors as modulators of health and functional capacity with age. In Ageing, Health and Competence, pp. 65-86 [J. Schroots, editor]. Amsterdam: Elsevier Science Publications.

Heikkinen, E., Waters, W. E. \& Brzezinski, Z. J. (1983). The Elderly in 11 Countries. A Sociomedical Survey. Public Health in Europe no. 21. Copenhagen: WHO Regional Office for Europe.

Hughes, J. M., Smithers, G., Gay, C., Clarke, P. C., Smith, P., Lowe, C., Prentice, A., Bates, C., Whitelaw, M. \& Bingham, S. (1995). The British National Diet and Nutrition Survey of people aged 65 years or over: protocol and feasibility study. Proceedings of the Nutrition Society 54, 631-643.

Institute of Medicine (1990). The Second Fifty Years: Promoting Health and Preventing Disability. Washington DC: National Academy Press.

James, W. P. T., Ferro-Luzzi, A. \& Waterlow, J. C. (1988). Definition of chronic energy deficiency in adults. European Journal of Clinical Nutrition 42, 969-981.

Jette, A. M. \& Branch, L. G. (1981). The Framingham Disability Study: II. Physical disability among the aging. American Journal of Public Health 71, 1211-1215.

Jette, A. M., Branch, L. G. \& Berlin, J. (1990). Musculoskeletal impairments and physical disablement among the aged. Journal of Gerontology 45, M203-M208.

Jitapunkul, S., Kamolratanakul, P. \& Ebrahim, S. (1994). The meaning of activities of daily living in a Thai elderly population: development of a new index. Age and Ageing 23, 97-101.

Kallman, D. A., Plato, C. C. \& Tobin, J. D. (1990). The role of muscle loss in the age-related decline of grip strength: cross sectional and longitudinal perspectives. Journal of Gerontology 45, M82-M88.

Kane, R. A. \& Kane, R. L. (1981). Assessing the Elderly: A Practical Guide to Measurement. Lexington, Mass: Lexington Books.

Katz, S. C., Ford, A. B., Moskowitz, R. W., Jackson, B. A. \& Jaffe, M. W. (1963). Studies of illness in the aged. The index of ADL: A standardized measure of biological and psychosocial function. Journal of the American Medical Association 185, 914-919.

Katz, S. \& Stroud, M. W. (1989). Functional assessment in geriatrics: a review of progress and directions. Journal of the American Geriatrics Society 37, 267-271.

Kelly, P. L. \& Kroemer, K. H. E. (1990). Anthropometry of elderly: status and recommendations. Human Factors 32, 571-595.

Kinsella, K. \& Suzman, R. (1992). Demographic dimensions of population aging in developing countries. American Journal of Human Biology 4, 3-8.

Klidjian, J., Foster, K. J., Kammerling, R. M., Cooper, A. \& Karran, S. J. (1980). Relation of anthropometric and dynamometric variables to serious postoperative complications. British Medical Journal 281, 899-901. 
Law, M. \& Letts, L. (1989). A critical review of scales of activities of daily living. American Journal of Occupational Therapy 43, 522-528.

Lawton, M. P. \& Brody, E. M. (1969). Assessment of older people: self-maintaining and instrumental activities of daily living. The Gerontologist 9, 179-186.

Lehmann, A. B. (1989). Review: undernutrition in elderly people. Age and Ageing 18, 339-353.

Liang, M. H. \& Jette, A. M. (1981). Measuring functional ability in chronic arthritis: a critical review. Arthritis and Rheumatism 24, 80-86.

Little, A., Hemsley, D., Bergmann, K., Volans, J. \& Levy, R. (1987). Comparison of the sensitivity of three instruments for the detection of cognitive decline in elderly living at home. British Journal of Psychiatry 150, 808-814.

Mansell, P. I., Rawlings, J., Allison, S. P., Bendall, M. J., Pearson, J., Bassey, E. J. \& Bastow, M. D. (1990). Low anthropometric indices in elderly females with fractured neck of femur. Clinical Nutrition 9, $190-194$.

Martin, S., Neale, G. \& Elia, M. (1985). Factors affecting maximal momentary grip strength. Human Nutrition: Clinical Nutrition 39C, 137-147.

Mowat, E. A., Thomas, S., Hyatt, R., Maxwell, J. D. \& Whitelaw, M. N. (1992). A comparison of nutritional intake, functional status and muscle strength between elderly day hospital and day centre attenders in South London. Journal of Human Nutrition and Dietetics 5, 35-51.

Mowé, M., Bohmer, T. \& Kindt, E. (1994). Reduced nutritional status in an elderly population is probable before disease and possibly contributes to the development of disease. American Journal of Clinical Nutrition 59, 317-324.

Nevitt, M. C., Cummings, S. R., Kidd, S. \& Black, D. (1989). Risk factors for recurrent nonsyncopal falls: a prospective study. Journal of the American Medical Association 261, 2663-2668.

Ostwald, S. K., Snowdon, D. A., Rysavy, S. D. M., Keenan, N. L. \& Kane, R. L. (1989). Manual dexterity as a correlate of dependency in the elderly. Journal of the American Geriatrics Society 37, 963-969.

Panton, L. B., Graves, J. E., Pollock, M. L., Hagberg, J. M. \& Chen, W. (1990). Effect of aerobic and resistance training on fractionated reaction time and speed of movement. Journal of Gerontology 45, M26-M31.

Payette, H. (1994). Nutritional status of community-residing elderly with functional limitations. Age and Nutrition 5, 4-7.

Phillips, S. K., Bruce, S. A., Newton, D. \& Woledge, R. C. (1992). The weakness of old age is not due to failure of muscle activation. Journal of Gerontology 47, M45-M49.

Pinholt, E. M., Kroenke, K., Hanley, J. F., Kussman, M. J., Twymann, P. L. \& Carpenter, J. L. (1987). Functional assessment of the elderly: a comparison of standard instruments with clinical judgement. Archives of Internal Medicine 147, 484-488.

Podsiadlo, D. \& Richardson, S. (1991). The timed 'up and go': a test of basic functional mobility for frail elderly persons. Journal of the American Geriatrics Society 39, 142-148.

Rantanen, T., Era, P. \& Heikkinen, E. (1994). Maximal isometric strength and mobility among 75 year old men and women. Age and Ageing 23, 132-137.

Reuben, D. B. \& Siu, A. L. (1990). An objective measure of physical function of elderly outpatients: the physical performance test. Journal of the American Geriatrics Society 38, 1105-1112.

Reuben, D. B., Siu, A. L. \& Kimpau, S. (1992). The predictive validity of self-report and performance-based measures of function and health. Journal of Gerontology 47, M106-M110.

Rikli, R. \& Busch, S. (1986). Motor performance of women as a function of age and physical activity level. Journal of Gerontology 41, 645-649.

Rodgers, W. L. \& Herzog, A. R. (1987). Interviewing older adults; the accuracy of factual information. Joumal of Gerontology 42, 387-394.

Roe, D. A. (1986). Nutritional assessment of the elderly. World Review of Nutrition and Dietetics 48, 85-113.

Rosenberg, I. H. \& Miller, J. W. (1992). Nutritional factors in physical and cognitive functions of elderly people. American Journal of Clinical Nutrition 55, 1237S-1243S.

Rutherford, O. M. \& Jones, D. A. (1992). The relationship of muscle and bone loss and activity levels with age in women. Age and Ageing 21, 286-293.

Seeman, T. E., Charpentier, P. A., Berkman, L. F., Tinetti, M. E., Guralnik, J. M., Albert, M., Blazer, D. \& Rowe, J. W. (1994). Predicting changes in physical performance in a high-functioning elderly cohort: MacArthur Studies of successful aging. Journal of Gerontology 49, M97-M108.

Shah, S., Vanclay, F. \& Cooper, B. (1989). Improving the sensitivity of the Barthel Index for stroke rehabilitation. Journal of Clinical Epidemiology 42, 703-709. 
Shizgall, H. M., Vasilevsky, C. A., Gardiner, P. F., Wang, W., Tuitt, D. A. Q. \& Brabant, G. V. (1986). Nutritional assessment and skeletal muscle function. American Journal of Clinical Nutrition 44, 761-771.

Siu, A. L., Hays, R. D., Ouslander, J. G., Osterwell, D., Valdez, R. B., Krynski, M. \& Gross, A. (1993). Measuring functioning and health in the very old. Journal of Gerontology 48, M10-M14.

Skelton, D. A., Greig, C. A., Davies, J. M. \& Young, A. (1994). Strength, power and related functional ability of healthy people aged 65-89 years. Age and Ageing 23, 371-377.

Solomons, N. W. (1992). Nutrition and aging: potentials and problems for research in developing countries. Nutrition Reviews 50, 224-229.

Spector, W. D., Katz, S., Murphy, J. B. \& Fulton, J. P. (1987). The hierarchical relationship between activities of daily living and instrumental activities of daily living. Journal of Chronic Diseases $\mathbf{4 0 , 4 8 1 - 4 8 9 .}$

Spirduso, W. W. (1980). Physical fitness, aging and psychomotor speed: a review. Journal of Gerontology 35, $850-865$.

Spitzer, W. D. (1987). State of science 1986: Quality of life and functional status as target variables for research. Journal of Chronic Disease 40, 465-471.

Sullivan, D. H., Patch, G. A., Walls, R. C. \& Lipschitz, D. A. (1990). Impact of nutritional status on morbidity and mortality in a select population of geriatric rehabilitation patients. American Journal of Clinical Nutrition 51, 749-758.

Syryani, L. K., Adnjana, A. K., Thong, D., Manik, I. R., Putra, D. K. W., Widjaja, W., Tama, D. W. \& Jensen, G. D. (1988). The physical and mental health of elderly in a Balinese village. Journal of Cross-Cultural Gerontology 3, 105-120.

Tinetti, M. E. (1986). Performance-orientated assessment of mobility problems in elderly patients. Journal of the American Geriatrics Society 34, 119-126.

Tinetti, M. E., Baker, D. I., McAvay, G., Claus, E. B., Garrett, P., Gottschalk, M., Koch, M. L., Trainor, K. \& Horwitz, R. I. (1994). A multifactorial intervention to reduce the risk of falling among elderly people living in the community. New England Journal of Medicine 331, 821-827.

Vellas, B. J., Garry, P. J. \& Abarede, J. L. (1992). Diseases and aging: patterns of morbidity with age, relationships between aging and age-associated diseases. American Journal of Clinical Nutrition 55, 1225S-1230S.

Wahlquist, M. L., Lukito, W. \& Hsu-Hage, B. H.-H. (1994). Nutrition and ageing in development. In Nutrition in the Nineties: Policy Issues, pp. 82-108 [M. R. Biswas and M. Gabr, editors]. New Delhi: Oxford University Press and WHO.

Wallace, R. B. \& Rohrer, J. E. (1990). Aging, quantitative health status assessment and the effectiveness of medical care. In Improving the Health of Older People: A World View, pp. 91-106 [R. L. Kane, J. Grimley Evans and D. Macfayden, editors]. New York: Oxford University Press and WHO.

Webb, A. R., Newman, L. A., Taylor, M. \& Keogh, J. B. (1989). Hand grip dynamometry as a predictor of postoperative complications reappraisal using age standardized grip strengths. Journal of Parenteral and Enteral Nutrition 13, 30-33.

Weiner, J. M., Hanley, R. J., Clark, R. \& van Nostrand, J. F. (1990). Measuring activities of daily living: comparisons across national surveys. Journal of Gerontology 45, S229-S237.

Wickham, C., Cooper, C., Margetts, B. M. \& Barker, D. J. P. (1989). Muscle strength, activity, housing and the risk of falls in elderly people. Age and Ageing 18, 47-51.

Williams, M. E., Gaylord, S. A. \& Gerrity, M. S. (1994). The timed manual performance test as a predictor of hospitalization and death in a community-based elderly population. Journal of the American Geriatrics Society 42, 21-27.

Williams, M. E., Hadler, N. M. \& Earp, J. A. L. (1982). Manual ability as a marker of dependency in geriatric women. Journal of Chronic Diseases 35, 115-122.

Wolfson, L. I. (1992). Gait and mobility. In Oxford Textbook of Geriatric Medicine, pp. $585-593$ [J. Grimley Evans and T. Franklin Williams, editors]. Oxford: Oxford University Press.

World Bank (1994). Averting the Old Age Crisis. New York: Oxford University Press, Inc.

Young, A. (1986). Exercise physiology in geriatric practice. Acta Medica Scandinavica 711, 227-232.

Young, A. (1992). Strength and power. In Oxford Textbook of Geriatric Medicine, pp. 597-604 [J. Grimley Evans and T. Franklin-Williams, editors]. Oxford: Oxford University Press. 